\title{
INFRARED OBSERVATIONS OF GALAXY CLUSTERS
}

\author{
D. ELBAZ \\ CEA - Service d'Astrophysique \\ Orme des Merisiers, 91191 Gif-sur-Yvette Cedex - France
}

\section{Introduction}

The evolution of galaxy clusters from their formation due to the merging of sub structures, the bulk of star formation and subsequent chemical enrichment of the intra-cluster medium, is expected to be quite recent $(\mathrm{z}<1-2)$ in the hierarchical clustering scenario (White \& Frenk 1991).

Some evidence for enhanced star formation at $\mathrm{z} \simeq 0.3-0.5$ has indeed been collected during the past ten years:

- excess of faint blue galaxies in the field (Tyson 1988) and in cluster galaxies (Butcher \& Oemler 1978).

- post-starburst or E+A galaxies in field and cluster galaxies (Zabludoff et al. 1996)

- steep rise in the global star formation rate of galaxies from $\mathrm{z}=0$ to 1 (Lilly et al. 1997)

However evidence is growing for the presence of well evolved rich clusters and cluster galaxies at high redshift (above $z=0.5-1$; Dickinson 1997, Mushotzky and Loewenstein 1997), which sets a key constraint on galaxy evolution models and on structure formation models as well.

After IRAS, which proved that starbursts emitted mostly in the infrared (Sanders \& Mirabel 1996), ISOCAM is finding a strong excess of faint mid-IR (12-18 $\mu \mathrm{m})$ emitting galaxies (Cesarsky 1997), demonstrating that dust may affect our view of the star formation history of the universe. This is confirmed at large redshifts by the effect of absorption in U and B drop-out selected galaxies (Pettini et al. 1997). The fact that galaxy interaction plays a dominant role both in the hierarchical clustering scenario and in IRAS ultra-luminous galaxies, strengthens the interest of studying the effect of the environment on the evolution of cluster galaxies, whereas the presence of dust requires the use of infrared telescopes like ISO to complete the analysis.

Observations of galaxy clusters can also set strong constraints on the evolution of galaxies through the analysis of intra-cluster iron (Elbaz et al. 1995), but also on diffuse dust emission outside galaxies, which could give real-time information on the current status of the cluster galaxies which would necessarily be replanishing dust (see below).

\section{Intracluster dust}

Wise et al. (1993) studied 56 clusters with IRAS and found two candidate detections of diffuse emission at $60 \mu \mathrm{m}$. Additional new evidence for the presence of dust outside galaxies has recently been found by Stickel et al. (1997), with the detection of an extended far infrared excess at 120 $\mu m$ at the center of the Coma cluster. This detection was done using the four pixel camera of ISOPHOT, C200, on-board ISO, with two 48 arcmin scans rotated about $50^{\circ}$, each done at 120 $\mu m$ and $185 \mu m$.

The authors interpret this excess as thermal emission from intracluster dust for a total dust mass of $0.6-16 \times 10^{8} M_{\odot}$. This extragalactic dust must be replenished continuously since dust is easily destroyed by the hot electrons of the intracluster medium through sputtering with a timescale of a few 10 million years. It is difficult, however, to see how the dust can be reinjected at a sufficiently fast rate, although three processes can be invoked: galactic winds, ram pressure stripping or the recent accretion of external material by the cluster. In the case of Coma a poor group of galaxies may have recently merged with the cluster in its central region. 
Because of the possible confusion with the smoothed emission of individual galaxies, this result would require confirmation with other clusters. But if confirmed, this result would set strong constraints on the recent evolution of galaxy clusters.

\section{Mid-Infrared Emission of Galaxies}

The following sections will present results obtained in the mid-infrared using ISOCAM on-board ISO, in the main broad-band filters of ISOCAM: LW2 $(5-8.5 \mu \mathrm{m})$ centered on $6.75 \mu \mathrm{m}$ and LW3 $(12-18 \mu \mathrm{m})$ centered on $15 \mu \mathrm{m}$. The rest frame mid-IR emission of galaxies can be divided into three components (Vigroux 1997, Puget 1997):

1. "carbon composites": produce the Unidentified Infrared Bands (UIBs) at 6.2, 7.7, 8.6, 11.3 and $12.7 \mu \mathrm{m}$ (the latest was discovered by ISO) as well as their underlying continuum.

2. Warm dust $(\mathrm{T}>150 \mathrm{~K})$ : thermal continuum at $\lambda>10 \mu \mathrm{m}$ from Very Small Grains (VSGs) of dust.

3. Forbidden lines of ionized gas: NeII $(12.8 \mu m)$, NeIII $(15.6 \mu m)$, SIV $(10.5 \mu m)$, ArII $(7 \mu m)$.

Only the first component affects the LW2 band, but all three components emit in the LW3 band, although only HII regions, where the UV spectrum is hard enough, exhibit strong ionized lines. As the galaxy redshift increases, LW3 becomes dominated by the first component while LW2 traces the old stellar component, due to K-correction.

\section{Star Formation in Nearby Clusters}

Boselli et al. (1997) have produced a statistical analysis of the mid-infrared properties of 117 late-type and $\mathrm{SO} / \mathrm{a}$ galaxies in the Virgo (99 galaxies detected) and Coma (18 galaxies detected) clusters, at $6.75 \mu \mathrm{m}$ (87 galaxies detected) and $15 \mu \mathrm{m}$ (72 detections). They used an additionnal sample of ellipticals to estimate the stellar contribution at 6.75 and $15 \mu \mathrm{m}$ normalized to the $\mathrm{K}$ ' flux (consistent with a simple black-body) and substracted it in the sample of late-types to keep only the dust contribution to the mid-IR emission. This residual emission originating from dust nicely correlates with the UV emission of the galaxies at $2000 \AA$, which is a good indicator of star formation. Hence, the rest-frame 6.75 and $15 \mu \mathrm{m}$ emission can be associated with star formation.

\section{Star Formation in $z=0.2$ Galaxy clusters}

At this redshift, K-correction does not change the physical components contributing to the LW2 and LW3 ISOCAM filters, which are therefore equivalent to rest-frame. A first result of the observation of A1732 ( $\mathrm{z}=0.193$, Pierre et al. 1997) is that mid-IR emitters avoid the cluster center, like galaxies providing the blue excess in the Butcher-Oemler effect, and appear to be morphologically disturbed.

Among 10 galaxies detected at $7 \mu \mathrm{m}$ and probably cluster members, only 2 were detected at 15 $\mu m$, but with a high 15 over $7 \mu m$ ratio, hence clearly associated with enhanced star formation. In A1689 $(z=0.18)$, the same excess is found ( 6 among 9 detections at $15 \mu \mathrm{m}$ show a high 15 over 7 $\mu m$ ratio). More than 20 galaxies were detected in each cluster at $7 \mu \mathrm{m}$, where ISOCAM is more sensitive. It is striking that none of the galaxies showing a clear excess of star formation in the mid-IR can be distinguished from the rest of the cluster galaxies from its optical colours, e.g. they do not show any particular blue or red excess. This effect is confirmed at larger redshift with the galaxies of the Hubble Deep Field detected by ISO (Aussel 1997), which are spread all over the colour-colour diagram, confirming that star formation is only partially sampled in the optical.

\section{Star Formation in $\mathrm{z}>0.4$ Galaxy clusters}

The K-correction becomes a dominant factor at redshifts above $\mathrm{z} \simeq 0.4$. The rest-frame emission coming from old stars will be shifted to the $7 \mu \mathrm{m}$ band, whereas the emission due to UIBs and their associated continuum, will fall in the $15 \mu \mathrm{m}$ band.

Six clusters were observed at these redshifts (3C295, 3C330, 0016+16, J1888, GHO1322, GHO1603) in the frame of a program looking for the evolution of cluster galaxies as a function of redshift (P.I. A.Franceschini). Most galaxies were detected at $7 \mu \mathrm{m}$, hence showing no clear excess of star formation, but the observations are not complete at faint fluxes (below $\simeq 1 \mathrm{mJy}$ ). Deeper integrations have therefore been scheduled. 
A search for distant galaxy clusters in the line of sight of bright quasars (PC1643+4631, Q0000263 ) is also in the process of being reduced (P.I. F.Mirabel).

\section{Conclusions and Perspectives}

ISOCAM deep surveys have been very fruitful, showing a clear excess of mid-IR emitting galaxies (Deep Survey in the Lockman Hole, Cesarsky 1997, and in the Hubble Deep Field, HDF, Aussel et al. 1997). These galaxies only show up at faint fluxes (below $\simeq 1 \mathrm{mJy}$ ) and the observations done at this stage on distant clusters are too shallow to allow any statistical analysis of such a population. However, the absence of any correlation between optical colours and mid-IR fluxes for galaxies in two nearby clusters (A1689 and $\mathrm{A} 1732$, at $\mathrm{z} \simeq 0.2$ ) is interesting since it could show that ISOCAM is revealing a different population of objects from those selected in the optical.

The comparison of $z \simeq 0.5-1$ galaxies in the field and in clusters will be allowed by the next observations in preparation. The sample of galaxies detected by ISOCAM within the HDF is already very promising since they also show no clear optical signature (like a blue excess) and that they lie above the no-evolution extrapolation from the IRAS counts.

\section{References}

Aussel, H., Elbaz, D., Starck, J.L., Cesarsky, C.J. (1997) Observation of the Hubble Deep Field with ISOCAM Application of the Preti Method, Extragalactic Astronomy in the Infrared-XXXIInd Moriond Meeting, Eds. G.A. Mamon, Trinh Xuan Thuan, and J. Tran Thanh Van, Ed. Frontieres, to appear

Boselli, A., et al. (1997) Mid-IR Colors and Star Formation in Virgo and Coma Galaxies, A \&jA, 324L, 13

Butcher, H., Oemler, A. JR. (1984) The evolution of galaxies in clusters. V - A study of populations since z approximately equal to $0.5, A p J, 285,426$

Cesarsky, C.J. (1997) Highlights from ISO: the Isocam Camera this issue

Deltorn, J. -M.; Lefevre, O., Crampton, D., Dickinson, M. (1997) A Massive Cluster of Galaxies at z $=0.996, A p J$, $483 \mathrm{~L}, 21$

Dickinson, M. (1997) Clusters of Galaxies at $\mathrm{z}>1$ HST and the High Redshift Universe, proceedings of the 37 th Herstmonceux Meeting, Eds. N. Tanvir, A. Aragon-Salamanca, and J.V. Wall, Ed. World Scientific, p.207

Elbaz, D., Arnaud, M., Vangioni-Flam, E. (1995) Bimodal Star Formation in Elliptical Galaxies and the Enrichment of the Intra-Cluster Medium, $A \& A, \mathbf{3 0 3}, 345$

Lilly, S.J., Lefevre, O., Hammer, F., Crampton, D. (1996) The Canada-France Redshift Survey: The Luminosity Density and Star Formation History of the Universe to z approximately $1, A p J, 460 \mathrm{~L}, 1$

Mushotzky, R.F., Loewenstein, M. (1997) Lack of Evolution in the Iron Abundance in Clusters of Galaxies and Implications for the Global Star Formation Rate at High Redshift, $A p J, 481 \mathrm{~L}, 63$

Pettini, M., et al. (1997) The Discovery of Primeval Galaxies and the Epoch of Galaxy Formation, astro-ph/9708117

Pierre, M., et al. (1997) A first glimpse into ISM/ICM connections at $\mathrm{z}=0.2$ with ISOCAM, $A B A, 315 \mathrm{~L}, 297$

Puget, J.L. (1998) Interstellar Matter, this issue

Sanders, D., Mirabel, I.F. (1996) Luminous Infrared Galaxies, ARAA, 34, 749

Stanford, S.A., Eisenhardt. P.R., Dickinson, M. (1997) The Evolution of Early-Type Galaxies in Distant Clusters, astro-ph/g708037

Stickel, M., Lemke, D., Mattila, K., Haikala, L.K., Haas, M. (1997) Far-Infrared Emission of Intracluster Dust in the Coma Galaxy Cluster, $A B A$, in press

Vigroux, L. (1997) ISO Observations of Normal Galaxies, Extragalactic Astronomy in the Infrared-XXXIInd Moriond Meeting, Eds. G.A. Mamon, Trinh Xuan Thuan, and J. Tran Thanh Van, Ed. Frontieres, to appear

White, S.D.M, Frenk, C.S. (1991) Galaxy Formation Through Hierarchical Clustering, $A p J, 379,52$

Wise, M.W, O'Connell, R.W., Bregman, J.N., Roberts, M.S. (1993) Far-Infrared Emission from the Intracluster Medium, $A p J, 405,94$

Zabludoff, A. et al. (1996) The Environment of "E+A" Galaxies, ApJ, 466, 104 